\title{
NON-TORSION NON-ALGEBRAIC CLASSES IN THE BROWN-PETERSON TOWER
}

\author{
MASAKI KAMEKO
}

\begin{abstract}
Generalizing the classical work of Atiyah and Hirzebruch on nonalgebraic classes, recently Quick proved the existence of torsion non-algebraic elements in the Brown-Peterson tower. We construct non-torsion non-algebraic elements in the Brown-Peterson tower for the prime number 2.
\end{abstract}

\section{INTRODUCTION}

Let $X$ be a smooth complex algebraic variety. We denote the space of complex points of $X$ with the analytic topology by $X(\mathbb{C})$. The Chow group $C H^{i} X$, the group generated by finite linear combinations of closed subvarieties of $X$ of codimension $i$ modulo rational equivalence, is a fundamental object to study in algebraic geometry. The cycle map from the Chow group $C H^{i} X$ to the singular cohomology $H^{2 i}(X(\mathbb{C}) ; \mathbb{Z})$ is also very important. We call an element in the cohomology of $X(\mathbb{C})$ algebraic if it is in the image of the cycle map. One of the most outstanding open problems concerning the cycle map is the Hodge conjecture. The Hodge conjecture asserts that for a projective smooth complex algebraic variety $X$, the cycle map

$$
C H^{i} X \otimes \mathbb{Q} \rightarrow\left\{\text { Hodge classes in } H^{2 i}(X(\mathbb{C}) ; \mathbb{Q})\right\}
$$

is surjective. In [Tot97, Totaro defined the Chow group $C H^{i} B G$ of the classifying space of a complex linear algebraic group $G$ by approximating it by quasi-projective smooth complex algebraic varieties and studied the cycle map

$$
\mathrm{cl}: C H^{i} B G \rightarrow H^{2 i}(B G ; \mathbb{Z}) \text {. }
$$

Let $p$ be a prime number and $(\mathbb{Z} / p)^{3}$ the elementary abelian $p$-group of rank 3 . In retrospect, in AH62, Atiyah and Hirzebruch used the non-surjectivity of the cycle map

$$
\text { cl: } C H^{2} B(\mathbb{Z} / p)^{3} \rightarrow H^{4}\left(B(\mathbb{Z} / p)^{3} ; \mathbb{Z}\right),
$$

that is, the existence of non-algebraic element in $H^{4}\left(B(\mathbb{Z} / p)^{3} ; \mathbb{Z}\right)$, to construct a projective smooth complex algebraic variety $X$ and a non-algebraic class in $H^{4}(X(\mathbb{C}) ; \mathbb{Z})$, that is a counterexample for the integral Hodge conjecture.

On the other hand, for each prime number $p$ and non-negative integer $m$, there exists the truncated Brown-Peterson cohomology $B P\langle m\rangle^{*}(X(\mathbb{C}))$ whose coefficient

2010 Mathematics Subject Classification. 14C25,19E15, 55T25.

Key words and phrases. Brown-Peterson cohomology, classifying space, algebraic cycle, cycle map.

This work was supported by JSPS KAKENHI Grant Numbers JP25400097 and JP17K05263. 
ring is $\mathbb{Z}_{(p)}\left[v_{1}, \ldots, v_{m}\right]$, where $\operatorname{deg} v_{i}=-2 p^{i}+2$. Also there exist natural transformations

$$
B P^{*}(X(\mathbb{C})) \stackrel{\rho_{m}}{\longrightarrow} B P\langle m\rangle^{*}(X(\mathbb{C}))
$$

and

$$
B P\langle n\rangle^{*}(X(\mathbb{C})) \stackrel{\rho_{m}^{n}}{\longrightarrow} B P\langle m\rangle^{*}(X(\mathbb{C})) \quad(n \geq m)
$$

such that $\rho_{m}\left(v_{i}\right)=0$ for $i>m, \rho_{m}\left(v_{i}\right)=v_{i}$ for $i \leq m$ and $\rho_{m}=\rho_{m}^{n} \circ \rho_{n}$. Moreover, there exist the motivic truncated Brown-Peterson cohomology $B P\langle m\rangle_{\mathcal{M}}^{*, *}(X)$ and the cycle map

$$
\mathrm{cl}: B P\langle m\rangle_{\mathcal{M}}^{2 i, i}(X) \rightarrow B P\langle m\rangle^{2 i}(X(\mathbb{C}))
$$

In the case $m=0$, the motivic truncated Brown-Peterson cohomology $B P\langle 0\rangle_{\mathcal{M}}^{*, *}(X)$ is nothing but the motivic cohomology $H_{\mathcal{M}}^{*, *}\left(X ; \mathbb{Z}_{(p)}\right)$, the Chow group localized at $p$, say $C H^{i} X_{(p)}$, is the motivic cohomology $H^{2 i, i}\left(X ; \mathbb{Z}_{(p)}\right)$ and the cycle map

$$
C H^{i} X_{(p)}=H_{\mathcal{M}}^{2 i, i}\left(X ; \mathbb{Z}_{(p)}\right)=B P\langle 0\rangle_{\mathcal{M}}^{2 i, i}(X) \rightarrow B P\langle 0\rangle^{2 i}(X(\mathbb{C}))=H^{2 i}\left(X(\mathbb{C}) ; \mathbb{Z}_{(p)}\right)
$$

is the classical cycle map. Recently, in Qui16, for each prime number $p$ and positive integer $m$, Quick proved the existence of a projective smooth complex algebraic variety $X$ and a non-algebraic element in $B P\langle m\rangle^{*}(X(\mathbb{C}))$, that is an element not in the image of the cycle map cl: $B P\langle m\rangle_{\mathcal{M}}^{2 i, i} X \rightarrow B P\langle m\rangle^{2 i}(X(\mathbb{C}))$. Quick uses the classifying space of the elementary abelian $p$-group $(\mathbb{Z} / p)^{m+3}$. So, Quick generalized the work of Atiyah and Hirzebruch to the Brown-Peterson tower. As examples of Atiyah and Hirzebruch, Quick's examples are also torsion classes.

One might wonder the existence of non-torsion examples. Counterexamples for the integral Hodge conjecture modulo torsion were obtained by Kollár. Kollár's examples are explained, for example, in SV05. In CTS10, Colliot-Thélène and Szamuely used the topological approach of Atiyah and Hirzebruch to give counterexamples for the integral Tate conjecture over finite fields and asked the existence of counterexamples for the integral Tate conjecture modulo torsion where Kollár's approach does not work. In PY15, counterexamples for the integral Hodge and Tate conjectures modulo torsion through the topological approach were given by Pirutka and Yagita using exceptional groups $G_{2}, F_{4}, E_{8}$ which contain elementary abelian $p$-subgroups $(\mathbb{Z} / p)^{3}$ for $p=2,3,5$, respectively. In Kam15, the author replaced the exceptional groups by $G_{1}=\left(S L_{p} \times S L_{p}\right) / \mu_{p}$ for all prime numbers $p$, where $S L_{p}$ is the special linear group over the complex numbers and $\mu_{p}$ is the center of $S L_{p}$ and $\mu_{p}$ acts on $S L_{p} \times S L_{p}$ diagonally.

In this paper, we construct non-torsion non-algebraic elements in the BrownPeterson tower for $p=2$ and for all positive integers $m$ by replacing the elementary abelian $p$-group $(\mathbb{Z} / p)^{m+3}$ by

$$
G_{m+1}=S L_{p}{ }^{m+2} / \mu_{p}
$$

the central quotient of product of special linear groups $S L_{p}$ where $\mu_{p}$ is the cyclic group of order $p$ and it acts on $S L_{p}{ }^{m+2}$ diagonally. We use the mod $p$ cohomology of the classifying space of $G_{n}$ to compute the Atiyah-Hirzebruch spectral sequence converging to the truncated Brown-Peterson cohomology $B P\langle m\rangle^{*}\left(B G_{n}\right)$. It is easy to compute the $\bmod p$ cohomology of $G_{n}$ for $p=2$ but it is not easy for odd prime numbers $p$. Hence our result is limited to the case $p=2$ although it is plausible that the similar results hold for all prime numbers $p$. 
Theorem 1.1. Suppose that $p=2$. For each non-negative integer $m$, there exists a non-torsion element $u$ in the integral cohomology of $B G_{m+1}$ such that the element $u$ lifts up to $B P\langle m\rangle^{*}\left(B G_{m+1}\right)$ but not to $B P\langle m+1\rangle^{*}\left(B G_{m+1}\right)$.

To state our result, Corollary [1.3, obtained from Theorem 1.1, let $\mathbb{G}_{m}$ be the multiplicative group of the non-zero complex numbers. For a reductive complex linear algebraic group $G$, we may approximate the classifying space $B\left(\mathbb{G}_{m} \times G\right)$ by projective smooth complex algebraic varieties. That is, for each positive integer $k$, there exists a projective smooth complex algebraic variety $X$ and a morphism of algebraic varieties that induces a continuous map $f: X(\mathbb{C}) \rightarrow B\left(\mathbb{G}_{m} \times G\right)$ which is a $k$-equivalence. In particular,

$$
f^{*}: H^{i}\left(B G ; \mathbb{Z}_{(p)}\right) \rightarrow H^{i}\left(X(\mathbb{C}) ; \mathbb{Z}_{(p)}\right)
$$

is an isomorphism for $i<k$. For the approximation of the above classifying space by projective smooth complex algebraic varieties, we refer the reader to Pirutka and Yagita PY15] and Antieau [Ant16 for the detail. For the motivic BrownPeterson cohomology $B P\langle m\rangle_{\mathcal{M}}^{2 i, i}(X)$, we refer the reader to Quick Qui16 for the detail. With these preparations, we have Corollary 1.3 on the existence of nontorsion non-algebraic classes of projective smooth complex algebraic varieties from Theorem [1.1. But, before we state Corollary 1.3, we show that, rationally, all cohomology classes obtained from the classifying space of a complex linear algebraic group are algebraic.

Proposition 1.2. Let $G$ be a complex linear algebraic group. Let $X$ be a projective smooth complex algebraic variety. Suppose that $f: X(\mathbb{C}) \rightarrow B G$ is the map induced by a morphism of complex algebraic varieties from $X$ to $B G$ and $v^{\prime} \in B P\langle m\rangle^{2 i}(B G)$. Then, there exists a nonzero integer $\alpha$ such that $\alpha f^{*}\left(v^{\prime}\right)$ is algebraic in the sense that $\alpha f^{*}\left(v^{\prime}\right)$ is in the image of the cycle map

$$
\mathrm{cl}: B P\langle m\rangle_{\mathcal{M}}^{2 i, i}(X) \rightarrow B P\langle m\rangle^{2 i}(X(\mathbb{C})) .
$$

Proof. First, we recall that

$$
B P_{\mathcal{M}}^{2 i, i}\left(B G L_{\ell}\right) \rightarrow B P^{2 i}\left(B G L_{\ell}\right)
$$

is an isomorphism Yag05, Proposition 9.1] and

$$
\rho_{m}: B P^{2 i}\left(B G L_{\ell}\right) \rightarrow B P\langle m\rangle^{2 i}\left(B G L_{\ell}\right)
$$

is surjective for the complex general linear group $G L_{\ell}$. Therefore, Chern classes in $B P\langle m\rangle^{*}(B G)$ of complex representations of $G$ are algebraic. Next, we recall that the rational cohomology $H^{*}(B G ; \mathbb{Q})$ is generated by Chern classes of complex representations of $G$ Lan72, Proof of Theorem 1]. The rational cohomology of $B G$ is a polynomial algebra generated by even degree elements $y_{1}, \ldots, y_{n}$. The AtiyahHirzebruch spectral sequence for $B P\langle m\rangle^{*}(B G) \otimes \mathbb{Q}$ collapses at the $E_{2}$-level and

$$
B P\langle m\rangle^{*}(B G) \otimes \mathbb{Q}=B P\langle m\rangle^{*}\left[\left[y_{1}, \ldots, y_{n}\right]\right] \otimes \mathbb{Q} .
$$

We may assume $y_{1}, \ldots, y_{n}$ are Chern classes of complex representations of $G$, so that $y_{1}, \ldots, y_{n}$ are elements in $B P\langle m\rangle^{*}(B G)$ and they are algebraic. Let

$$
j: B P\langle m\rangle^{2 i}(B G) \rightarrow B P\langle m\rangle^{2 i}(B G) \otimes \mathbb{Q}
$$

be the homomorphism induced by the inclusion map $\mathbb{Z}_{(p)} \rightarrow \mathbb{Q}$. Then,

$$
j\left(v^{\prime}\right)=\sum_{I, J} \frac{\beta_{I J}}{\alpha_{I J}} v_{I} y_{J}
$$


where $v_{I}$ ranges over monomials in $v_{1}, \ldots, v_{m}, y_{J}$ ranges over monomials in $y_{1}, \ldots, y_{n}$, under the restriction

$$
\operatorname{deg} v_{I}+\operatorname{deg} y_{J}=2 i,
$$

$\alpha_{I J}$ are nonzero integers and $\beta_{I J}$ are integers. The element $j\left(v^{\prime}\right)$ is an infinite linear combination of such $v_{I} y_{J}$. Let $\operatorname{dim} X(\mathbb{C})$ be the dimension of $X(\mathbb{C})$ as a differentiable manifold. Since there exist only finite number of $v_{I} y_{J}$ such that

$$
\operatorname{deg} v_{I}+\operatorname{deg} y_{J}=2 i, \quad \operatorname{deg} y_{J} \leq \operatorname{dim} X(\mathbb{C}),
$$

we may choose a common multiplier $\alpha^{\prime \prime}$ of $\alpha_{I J}$ of such $(I, J)$. Let us define the element $v^{\prime \prime}$ in $B P\langle m\rangle^{2 i}(B G)$ by

$$
v^{\prime \prime}=\sum_{I, J} \frac{\alpha^{\prime \prime} \beta_{I J}}{\alpha_{I J}} v_{I} y_{J}
$$

where $\operatorname{deg} v_{I}+\operatorname{deg} y_{J}=2 i, \operatorname{deg} y_{J} \leq \operatorname{dim} X(\mathbb{C})$. Then, $v^{\prime \prime}$ is algebraic and

$$
j\left(\alpha^{\prime \prime} v^{\prime}-v^{\prime \prime}\right)=\sum_{I, J} \frac{\alpha^{\prime \prime} \beta_{I J}}{\alpha_{I J}} v_{I} y_{J}
$$

where $\operatorname{deg} v_{I}+\operatorname{deg} y_{J}=2 i, \operatorname{deg} y_{J}>\operatorname{dim} X(\mathbb{C})$. Therefore, in $B P\langle m\rangle^{2 i}(X(\mathbb{C})) \otimes \mathbb{Q}$, we have

$$
j\left(f^{*}\left(\alpha^{\prime \prime} v^{\prime}-v^{\prime \prime}\right)\right)=0
$$

On the other hand, since $X(\mathbb{C})$ has the homotopy type of a finite complex, the kernel of

$$
j: B P\langle m\rangle^{2 i}(X(\mathbb{C})) \rightarrow B P\langle m\rangle^{2 i}(X(\mathbb{C})) \otimes \mathbb{Q}
$$

is torsion and there exists a nonzero integer $\alpha^{\prime}$ such that

$$
\alpha^{\prime}\left(\alpha^{\prime \prime} f^{*}\left(v^{\prime}\right)-f^{*}\left(v^{\prime \prime}\right)\right)=0 .
$$

So, putting $\alpha=\alpha^{\prime} \alpha^{\prime \prime}$, we have that

$$
\alpha f^{*}\left(v^{\prime}\right)=\alpha^{\prime} f^{*}\left(v^{\prime \prime}\right)
$$

and it is algebraic.

Now, we state and prove Corollary 1.3 on the existence of non-torsion, nonalgebraic classes assuming Theorem 1.1.

Corollary 1.3. Suppose that $p=2$. For each non-negative integer $m$, there exists a projective smooth complex algebraic variety $X$ and an element $v$ in $B P\langle m\rangle^{*}(X)$ such that

(1) $v$ is non-algebraic, that is, not in the image of the cycle map

$$
\mathrm{cl}: B P\langle m\rangle_{\mathcal{M}}^{2 i, i}(X) \rightarrow B P\langle m\rangle^{2 i}(X(\mathbb{C})),
$$

(2) there exists a nonzero integer $\alpha$ such that the scalar multiple $\alpha v$ of $v$ is algebraic, and

(3) $\rho_{0}^{m}(v)$ is a non-torsion class in $H^{2 i}\left(X(\mathbb{C}) ; \mathbb{Z}_{(p)}\right)$,

where $2 i=\operatorname{deg} v$. 
Proof. Let $G_{m+1}$ be the algebraic group $G_{m+1}$ in Theorem 1.1 and $u \in H^{2 i}\left(B G_{m+1} ; \mathbb{Z}_{(p)}\right)$ be the element $u$ in Theorem 1.1. For the sake of notational simplicity, let $G=$ $\mathbb{G}_{m} \times G_{m+1}$

By the Künneth theorem, we have

$$
H^{2 i}\left(B G ; \mathbb{Z}_{(p)}\right)=\bigoplus_{k} H^{k}\left(B \mathbb{G}_{m} ; \mathbb{Z}_{(p)}\right) \otimes H^{2 i-k}\left(B G_{m+1} ; \mathbb{Z}_{(p)}\right)
$$

Let us denote by $1 \otimes u$ the element in $H^{2 i}\left(B G ; \mathbb{Z}_{(p)}\right)$ corresponding to the element $1 \otimes u$ in

$$
H^{0}\left(B \mathbb{G}_{m} ; \mathbb{Z}_{(p)}\right) \otimes H^{2 i}\left(B G_{m+1} ; \mathbb{Z}_{(p)}\right) .
$$

In the Atiyah-Hirzebruch spectral sequence for $B P\langle m\rangle^{*}(B G)$,

$$
(1 \otimes u) \otimes 1 \in E_{2}^{2 i, 0}
$$

is a permanent cycle. Let $v^{\prime}$ be an element in $B P\langle m\rangle^{2 i}(B G)$ such that

$$
\rho_{0}^{m}\left(v^{\prime}\right)=1 \otimes u \text {. }
$$

In the Atiyah-Hirzebruch spectral sequence for $B P\langle m+1\rangle^{*}(B G)$, for some $r$,

$$
d_{r}((1 \otimes u) \otimes 1) \neq 0 \in E_{r}^{2 i+r, 1-r} .
$$

Choose a projective smooth complex algebraic variety $X$ and a morphism from $X$ to $B G$, so that

$$
f^{*}: H^{k}\left(B G ; \mathbb{Z}_{(p)}\right) \rightarrow H^{k}\left(X(\mathbb{C}) ; \mathbb{Z}_{(p)}\right)
$$

is an isomorphism for $k \leq 2 i+r$, where $f: X(\mathbb{C}) \rightarrow B G$ is the map induced by the morphism of algebraic varieties. Then, in the Atiyah-Hirzebruch spectral sequence for $B P\langle m+1\rangle^{*}(X(\mathbb{C}))$,

$$
d_{r}\left(f^{*}(1 \otimes u) \otimes 1\right) \neq 0 .
$$

Therefore, $f^{*}(1 \otimes u)$ is a non-torsion class and $f^{*}(1 \otimes u)$ is in the image of $\rho_{0}^{m}$ but not in the image of $\rho_{0}^{m+1}$.

Let $v=f^{*}\left(v^{\prime}\right)$. Then, $\rho_{0}^{m}(v)=f^{*}(1 \otimes u)$. So, the element $v$ satisfies the condition (3). By Proposition 1.2, the element $v$ satisfies the condition (2). As for the condition (1), we consider the following commutative diagram:

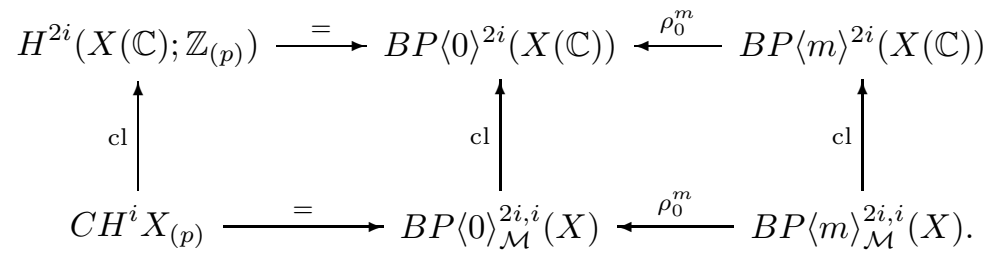

The image of the composition of $\rho_{0}^{m}$ and the cycle map

$$
\mathrm{cl}: B P\langle m\rangle_{\mathcal{M}}^{2 i, i}(X) \rightarrow B P\langle m\rangle^{2 i}(X(\mathbb{C})),
$$

is a subset of the image of the cycle map

$$
\text { cl: } C H^{i}(X) \rightarrow H^{2 i}\left(X(\mathbb{C}) ; \mathbb{Z}_{(p)}\right) .
$$

However, Totaro proved that the image of the cycle map is in the image of $\rho_{0}$. Thus, since $f^{*}(1 \otimes u)$ does not lift up to $B P\langle m+1\rangle^{2 i}(X(\mathbb{C}))$, the element $v$ is not in the image of the cycle map and it satisfies the condition (1). 
The Brown-Peterson cohomology plays an interesting role in both algebraic geometry and algebraic topology, especially in conjunction with the study of the cycle map. In [Tot97], Totaro showed that the cycle map

$$
\text { cl: } C H^{i} X_{(p)} \rightarrow H^{2 i}\left(X(\mathbb{C}) ; \mathbb{Z}_{(p)}\right)
$$

factors through $B P^{*}(X(\mathbb{C})) \otimes_{B P^{*}} \mathbb{Z}_{(p)}$. He also conjectured that the cycle map

$$
C H^{i} B G_{(p)} \rightarrow\left(B P^{*}(B G) \otimes_{B P^{*}} \mathbb{Z}_{(p)}\right)^{2 i}
$$

is an isomorphism for a complex linear algebraic group $G$ when the Brown-Peterson cohomology of $B G$ has no nonzero odd degree elements. The Brown-Peterson cohomology had been studied in algebraic topology long before Totaro's work. It is also conjectured in KY93, that the Brown-Peterson cohomology of the classifying space of a compact Lie group has no nonzero odd degree element. This conjecture also remains as an open problem and it is a precursor of Totaro's conjecture. Note that the maximal compact subgroup of a reductive complex linear algebraic group is a compact Lie group and they are homotopy equivalent to each other. So, the truncated Brown-Peterson cohomology theories of classifying spaces of these groups are the same to each other. In particular, $G_{n}$ 's maximal compact subgroup is $S U(p)^{n+1} / \mu_{p}$, where $S U(p)$ is the special unitary group.

In this paper, through the computation of the Atiyah-Hirzebruch spectral sequence, we give a new family of compact Lie groups $S U(2)^{n+1} / \mu_{2}$ for which the Brown-Peterson cohomology of the classifying space has no nonzero odd degree element.

Theorem 1.4. Suppose that $p=2, m$ is a non-negative integer and $n$ is a positive integer. The truncated Brown-Peterson cohomology $B P\langle m\rangle^{*} B G_{n}$ has no nonzero odd degree elements if and only if $m \geq n+1$. Moreover, the Brown-Peterson cohomology $B P^{*}\left(B G_{n}\right)$ has no nonzero odd degree elements and the natural transformation $\rho_{m}: B P^{*}\left(B G_{n}\right) \rightarrow B P\langle m\rangle^{*}\left(B G_{n}\right)$ is surjective if and only if $m \geq n+1$.

For $n=1, G_{1}=S O(4)$. The Brown-Peterson cohomology of $B G_{1}=B S O(4)$ is computed by Kono and Yagita [KY93, Theorem 5.5].

This paper is organized as follows: In Section 2, we compute the mod 2 cohomology of $B G_{n}$ and the action of the Milnor operations. In Section 3, we define a homomorphism $\phi_{r}$ and prove Lemmas 3.2 and 3.3. We need the homomorphism $\phi_{r}$ to state our main result. We use these lemmas in Sections 4 and 5. In Section 4, we write down the Atiyah-Hirzebruch spectral sequence for $B P\langle m\rangle^{*}\left(B G_{n}\right)$ as Propositions 4.1 and 4.2. In Section 5, we prove the results stated in Section 4 .

\section{The MOD 2 COHOMOLOGY RING}

In this section, we compute the mod 2 cohomology ring of $B G_{n}$ and the action of the Milnor operations on it.

First, we compute the mod 2 cohomology of $B G_{n}$ for $n \geq 1$. We consider the projection $\pi$ obtained by removing the $(n+1)$-th factor, that is,

$$
\pi: S L_{2}^{n+1} \rightarrow S L_{2}^{n}, \quad \pi\left(m_{0}, m_{1}, \ldots, m_{n}\right)=\left(m_{0}, m_{1}, \ldots, m_{n-1}\right) .
$$

It induces a fibre sequence

$$
B S L_{2} \longrightarrow B G_{n} \stackrel{\pi}{\longrightarrow} B G_{n-1} .
$$


It is clear that $G_{0}, B G_{0}$ are homotopy equivalent to $S O(3), B S O(3)$, respectively. Therefore, we have

$$
H^{*}\left(B G_{0} ; \mathbb{Z} / 2\right)=\mathbb{Z} / 2\left[x_{2}, x_{3}\right],
$$

where $x_{2}$ is the generator of $H^{2}(B S O(3) ; \mathbb{Z} / 2), x_{3}=Q_{0} x_{2}$ and $Q_{0}$ is the mod 2 Bockstein homomorphism. Let us recall the mod 2 cohomology of $B S L_{2}$. The $\bmod$ 2 cohomology of $B S L_{2}$ is a polynomial algebra generated by the single generator $c_{2}$ of degree 4 . The Leray-Serre spectral sequence associated with the above fibre sequence has the $E_{2}$-term isomorphic to

$$
H^{*}\left(B G_{n-1} ; \mathbb{Z} / 2\right) \otimes H^{*}\left(B S L_{2} ; \mathbb{Z} / 2\right) .
$$

The $E_{2}$-term is generated by $1 \otimes c_{2}$ as an algebra over $E_{2}^{*, 0}$. So, in order to prove that the spectral sequence collapses at the $E_{2}$-level, it suffices to show that $1 \otimes c_{2}$ is a permanent cycle. For dimensional reasons, $d_{r}\left(1 \otimes c_{2}\right)=0$ except for $r=5$ and $d_{r}\left(1 \otimes c_{2}\right) \in E_{5}^{5,0}$. Let us consider the map $\sigma: G_{n-1} \rightarrow G_{n}$ induced by $\sigma: S L_{2}^{n} \rightarrow S L_{2}{ }^{n+1}$, given by

$$
\sigma\left(m_{0}, \ldots, m_{n-1}\right)=\left(m_{0}, \ldots, m_{n-1}, m_{n-1}\right) .
$$

Then, $\pi \circ \sigma$ is the identity map. Hence, all elements in $E_{2}^{*, 0}$ are permanent cycles. Therefore, we have $d_{5}\left(1 \otimes c_{2}\right)=0$ and so $1 \otimes c_{2}$ is also a permanent cycle. So, the spectral sequence collapses at the $E_{2}$-level and we have

$$
H^{*}\left(B G_{n} ; \mathbb{Z} / 2\right)=H^{*}\left(B G_{n-1} ; \mathbb{Z} / 2\right) \otimes H^{*}\left(B S L_{2} ; \mathbb{Z} / 2\right)
$$

as an algebra over $H^{*}\left(B G_{n-1} ; \mathbb{Z} / 2\right)$. In particular, for $n=1$, we have

$$
H^{*}\left(B G_{1} ; \mathbb{Z} / 2\right)=\mathbb{Z} / 2\left[x_{2}, x_{3}, x_{4}\right]
$$

for some $x_{4}$. We make the choice of $x_{4}$ in due course. Inductively, we have

$$
H^{*}\left(B G_{n} ; \mathbb{Z} / 2\right)=\mathbb{Z} / 2\left[x_{2}, x_{3}, x_{41}, \ldots, x_{4 n}\right]
$$

for some degree 4 elements $x_{41}, \ldots, x_{4 n}$. For $i=1, \ldots, n$, let $\pi_{i}: G_{n} \rightarrow G_{1}$ be the map induced by the projection of $S L_{2}{ }^{n+1}$ to $S L_{2}{ }^{2}$ given by

$$
\left(m_{0}, \ldots, m_{n}\right) \mapsto\left(m_{0}, m_{i}\right) .
$$

Then, we have the following proposition.

Proposition 2.1. The mod 2 cohomology of $B G_{n}$ is a polynomial algebra over $\mathbb{Z} / 2$,

$$
\mathbb{Z} / 2\left[x_{2}, x_{3}, x_{41}, \ldots, x_{4 n}\right]
$$

where $\operatorname{deg} x_{2}=2, \operatorname{deg} x_{3}=3, \operatorname{deg} x_{4 i}=4$. The generator $x_{4 i}$ is $\pi_{i}^{*}\left(x_{4}\right)$.

Next, we consider the subgroup $(\mathbb{Z} / 2)^{n+2}$ of $G_{n}$. Let us consider elements

$$
I=\left(\begin{array}{ll}
1 & 0 \\
0 & 1
\end{array}\right), \xi=\left(\begin{array}{cc}
-1 & 0 \\
0 & -1
\end{array}\right), \eta=\left(\begin{array}{cc}
i & 0 \\
0 & -i
\end{array}\right), \zeta=\left(\begin{array}{ll}
0 & i \\
i & 0
\end{array}\right),
$$

in $S L_{2}$. Let

$$
\Delta: S L_{2} \rightarrow S L_{2}^{n+1}
$$

be the diagonal map, that is, $\Delta(m)=(m, \ldots, m)$. The subgroup $\mu_{2}$ of the center of $S L_{2}{ }^{n+1}$ is the subgroup generated by $\Delta(\xi)=(\xi, \ldots, \xi)$. For $i=0, \ldots, n$, let us write

$$
\Gamma_{i}: S L_{2} \rightarrow S L_{2}^{n+1}
$$


for the inclusion map into the $i$-th factor such that

$$
\Gamma_{i}(m)=\left(m_{0}, \ldots, m_{n}\right),
$$

where $m_{i}=m, m_{j}=I$ for $j \neq i$. Let

$$
\pi^{\prime}: S L_{2}{ }^{n+1} \rightarrow S L_{2}{ }^{n+1} / \mu_{2}=G_{n}
$$

be the obvious projection. Then, the elements

$$
\pi^{\prime}(\Delta(\eta)), \pi^{\prime}(\Delta(\zeta)), \pi^{\prime}\left(\Gamma_{i}(\xi)\right), \quad(i=1, \ldots, n),
$$

generate a subgroup isomorphic to the elementary abelian 2-group of rank $n+2$. We denote it by $(\mathbb{Z} / 2)^{n+2}$ and we denote the inclusion map of $(\mathbb{Z} / 2)^{n+2}$ into $G_{n}$ by

$$
\iota_{n}:(\mathbb{Z} / 2)^{n+2} \rightarrow G_{n} .
$$

Now, we recall some facts on the induced homomorphism

$$
\iota_{1}^{*}: H^{*}\left(B G_{1} ; \mathbb{Z} / 2\right) \rightarrow H^{*}\left(B(\mathbb{Z} / 2)^{3} ; \mathbb{Z} / 2\right) .
$$

We denote by $s_{1}, s_{2}, t$ generators of $H^{1}\left(B(\mathbb{Z} / 2)^{3} ; \mathbb{Z} / 2\right)$ corresponding to the generators $\pi^{\prime}(\Delta(\eta)), \pi^{\prime}(\Delta(\zeta)), \pi^{\prime}\left(\Gamma_{1}(\xi)\right)$. As above, let $x_{2}$ be the generator of $H^{2}\left(B G_{1} ; \mathbb{Z} / 2\right)=$ $\mathbb{Z} / 2$. Let $x_{3}=Q_{0}\left(x_{2}\right)$. Then,

$$
\iota_{1}^{*}\left(x_{2}\right)=s_{1}^{2}+s_{1} s_{2}+s_{2}^{2}, \quad \iota_{1}^{*}\left(x_{3}\right)=s_{1}^{2} s_{2}+s_{1} s_{2}^{2},
$$

respectively. The image of $\iota_{1}^{*}$ is generated by the above two elements and

$$
t^{4}+\left(s_{1}^{2}+s_{1} s_{2}+s_{2}^{2}\right) t^{2}+\left(s_{1}^{2} s_{2}+s_{1} s_{2}^{2}\right) t,
$$

as an algebra over $\mathbb{Z} / 2$. See $[$ Kam15, Section 6$]$, if needed, for the details of the image of generators of $H^{*}\left(B G_{1} ; \mathbb{Z} / 2\right)$ by the induced homomorphism $\iota_{1}^{*}$. We choose the generator $x_{4}$ of $H^{4}\left(B G_{1} ; \mathbb{Z} / 2\right)$, so that its image $\iota_{1}^{*}\left(x_{4}\right)$ is the above element of degree 4 . Then, it is easy to see that the following proposition holds.

Proposition 2.2. The induced homomorphism

$$
\iota_{n}^{*}: H^{*}\left(B G_{n} ; \mathbb{Z} / 2\right) \rightarrow H^{*}\left(B(\mathbb{Z} / 2)^{n+2} ; \mathbb{Z} / 2\right)
$$

is injective.

Finally, we close this section by determining the action of Milnor operations on $x_{3}, x_{4}$. It plays an important role in the computation of the differentials of the Atiyah-Hirzebruch spectral sequence for $B P\langle m\rangle^{*}\left(B G_{n}\right), n \geq 2$. Let us denote by $Q_{j}$ the Milnor operation of degree $2^{j+1}-1$.

Proposition 2.3. For $j \geq 1,0 \leq k \leq j-1$, there exist polynomials $\alpha_{j k}$ in $x_{2}^{2}, x_{3}^{2}$ such that

$$
Q_{j}\left(x_{3}\right)=\alpha_{j 0} x_{3}^{2}, \quad Q_{j}\left(x_{4}\right)=x_{3}\left(\sum_{k=0}^{j-1} \alpha_{j k} x_{4}^{2^{k}}\right)
$$

in $H^{*}\left(B G_{1} ; \mathbb{Z} / 2\right)=\mathbb{Z} / 2\left[x_{2}, x_{3}, x_{4}\right]$.

Proof. For the sake of notational simplicity, by abuse of notation, we denote $\iota_{1}^{*}\left(x_{i}\right)$ by $x_{i}$. By direct calculation, we have

$$
\begin{aligned}
& Q_{0}\left(x_{3}\right)=0, \quad Q_{1}\left(x_{3}\right)=x_{3}^{2}, \quad Q_{2}\left(x_{3}\right)=x_{2}^{2} x_{3}^{2}, \\
& Q_{0}\left(x_{4}\right)=0, \quad Q_{1}\left(x_{4}\right)=x_{3} x_{4}, \quad Q_{2}\left(x_{4}\right)=x_{3} x_{4}^{2}+x_{2}^{2} x_{3} x_{4} .
\end{aligned}
$$

We define $\alpha_{10}=0, \alpha_{20}=x_{2}^{2}, \alpha_{21}=1$ and the proposition holds for $i=1,2$. 
For $j \geq 2$, let

$$
\mathcal{O}_{j}=Q_{j}+x_{2}^{2^{j-1}} Q_{j-1}+x_{3}^{2^{j-1}} Q_{j-2} .
$$

It is clear that $\mathcal{O}_{j}$ acts as a derivation on both $H^{*}\left(B G_{1} ; \mathbb{Z} / 2\right)$ and $H^{*}\left(B(\mathbb{Z} / 2)^{3} ; \mathbb{Z} / 2\right)$ and

$$
\mathcal{O}_{2}\left(s_{1}\right)=\mathcal{O}_{2}\left(s_{2}\right)=0, \quad \mathcal{O}_{2}(t)=x_{4}^{2}
$$

It is also easy to verify that

$$
\mathcal{O}_{j}\left(s_{1}\right)=\left(\mathcal{O}_{2}\left(s_{1}\right)\right)^{2^{j-2}}, \quad \mathcal{O}_{j}\left(s_{2}\right)=\left(\mathcal{O}_{2}\left(s_{2}\right)\right)^{2^{j-2}}, \quad \mathcal{O}_{j}(t)=\left(\mathcal{O}_{2}(t)\right)^{2^{j-2}},
$$

for $j \geq 2$. Hence, for $j \geq 2$, in $H^{*}\left(B(\mathbb{Z} / 2)^{3} ; \mathbb{Z} / 2\right)$, we have

$$
\mathcal{O}_{j}\left(s_{1}\right)=\mathcal{O}_{j}\left(s_{2}\right)=0 \quad \text { and } \quad \mathcal{O}_{j}(t)=x_{4}^{2^{j-1}} .
$$

Since $\mathcal{O}_{j}$ is a derivation, we have

$$
\mathcal{O}_{j}\left(x_{3}\right)=0, \quad \mathcal{O}_{j}\left(x_{4}\right)=x_{3} \mathcal{O}_{j}(t)=x_{3} x_{4}^{2^{j-1}} .
$$

Therefore, we have

$$
\begin{aligned}
& Q_{j}\left(x_{3}\right)=x_{2}^{2^{j-1}} Q_{j-1} x_{3}+x_{3}^{2^{j-1}} Q_{j-2} x_{3}, \\
& Q_{j}\left(x_{4}\right)=x_{3} x_{4}^{2^{j-1}}+x_{2}^{2^{j-1}} Q_{j-1} x_{4}+x_{3}^{2^{j-1}} Q_{j-2} x_{4} .
\end{aligned}
$$

We prove the proposition by induction on $j$. Assume that $j \geq 3$ and the proposition holds for $j-1$ and $j-2$. Then, we have

$$
\begin{aligned}
Q_{j}\left(x_{3}\right) & =x_{2}^{2^{j-1}} Q_{j-1}\left(x_{3}\right)+x_{3}^{2^{j-1}} Q_{j-2}\left(x_{3}\right) \\
& =x_{3}\left(\alpha_{j-1,0} x_{2}^{2^{j-1}}+\alpha_{j-2,0} x_{3}^{2^{j-1}}\right),
\end{aligned}
$$

and

$$
\begin{aligned}
Q_{j}\left(x_{4}\right) & =x_{3} x_{4}^{2^{j-1}}+x_{2}^{2^{j-1}} Q_{j-1}\left(x_{4}\right)+x_{3}^{2^{j-1}} Q_{j-2}\left(x_{4}\right) \\
& =x_{3}\left(x_{4}^{2^{j-1}}+\alpha_{j-1, j-2} x_{2}^{2^{j-1}} x_{4}^{2^{j-2}}+\sum_{k=0}^{j-3}\left(\alpha_{j-1, k} x_{2}^{2^{j-1}}+\alpha_{j-2, k} x_{3}^{2^{j-1}}\right) x_{4}^{2^{k}}\right) .
\end{aligned}
$$

Hence, we have

$$
\begin{aligned}
\alpha_{j, j-1} & =1, \\
\alpha_{j, j-2} & =\alpha_{j-1, j-2} x_{2}^{2^{j-1}} \\
\alpha_{j, k} & =\alpha_{j-1, k} x_{2}^{2^{j-1}}+\alpha_{j-2, k} x_{3}^{2^{j-1}}
\end{aligned}
$$

for $0 \leq k<j-2$ as desired.

\section{LEMMAS}

Let $M_{n}=\mathbb{Z} / 2\left[x_{2}^{2}, x_{3}, x_{41}, \ldots, x_{4 n}\right]$ and we regard $M_{n}$ as a subalgebra of the mod 2 cohomology ring of $B G_{n}$ in Proposition 2.1. It is closed under the action of Milnor operations $Q_{j}$. In this section, we define a homomorphism $\phi_{r}: M_{n} \rightarrow M_{n}$. We use this $\phi_{r}$ in the statement of our main result, Proposition 4.2. Then, we prove Lemmas 3.2 and 3.3 on this homomorphism $\phi_{r}$. We use Lemma 3.2 in the proof of Theorem 1.4 in Section 4 and Lemma 3.3 in the proof of Proposition 4.2 in Section 5 respectively. We do not use Lemmas 3.2 and 3.3 elsewhere. 
We set up some notations. For a ring $R$ and a finite set $\{y\}$, we write $R\{y\}$ for the free $R$-module with the basis $\{y\}$. Let

$$
\begin{aligned}
M_{n} & =\mathbb{Z} / 2\left[x_{2}^{2}, x_{3}, x_{41}, \ldots, x_{4 n}\right], \\
L_{n} & =\mathbb{Z} / 2\left[x_{2}^{2}, x_{3}, x_{41}^{2}, \ldots, x_{4 n}^{2}\right],
\end{aligned}
$$

For a finite set $I=\left\{i_{1}, \ldots, i_{\ell}\right\}$ of positive integers such that $1 \leq i_{1}<\cdots<i_{\ell} \leq n$, we write $x_{I}$ for $x_{4 i_{1}} \cdots x_{4 i_{\ell}}$ and $\ell(I)$ for $\ell \geq 1$. It is clear that $M_{n}$ is a free $L_{n^{-}}$ module with the basis $\left\{1, x_{I}\right\}$. Let us denote by $M_{n, i}$ the submodule spanned by $\left\{x_{I} \mid \ell(I)=i\right\}$ for $i \geq 1$. We write $M_{n, 0}$ for $L_{n}\{1\} \subset M_{n}$. For $j \geq 2$, we define derivations $\partial_{i}$ by

$$
\begin{aligned}
\partial_{j}\left(x_{3}\right) & =0, \\
\partial_{j}\left(x_{4 i}\right) & =\sum_{k=1}^{j-1} \alpha_{j k} x_{4 i}^{2^{k}},
\end{aligned}
$$

where the coefficients $\alpha_{j k}$ are those in Proposition 2.3. From this definition, it is clear that

$$
Q_{j}\left(x_{3} x_{4 i}\right)=x_{3}^{2} \partial_{j}\left(x_{4 i}\right), \quad Q_{j}\left(x_{4 i_{1}} x_{4 i_{2}}\right)=x_{3} \partial_{j}\left(x_{4 i_{1}} x_{4 i_{2}}\right) .
$$

Moreover, $\partial_{j}$ is an $L_{n}$-module homomorphism from $M_{n, i+1}$ to $M_{n, i}$. We define an $L_{n}$-module homomorphism $\phi_{r}: M_{n, i+r-1} \rightarrow M_{n, i}$ by

$$
\phi_{r}(x)=\partial_{2} \cdots \partial_{r}(x) \in M_{n, i},
$$

where $x \in M_{n, i+r-1}$. The following proposition is immediate from the definition of $\phi_{r}$ and the fact that

$$
Q_{j}\left(x_{3} x_{4 i}\right)=x_{3}^{2} \partial_{j}\left(x_{4 i}\right), \quad Q_{j}\left(x_{4 i_{1}} x_{4 i_{2}}\right)=x_{3} \partial_{j}\left(x_{4 i_{1}} x_{4 i_{2}}\right) .
$$

Proposition 3.1. Suppose that $\ell(I)-r+1+s$ is even and $s \geq 0$. Then, we have

$$
Q_{r+1}\left(\phi_{r}\left(x_{I}\right) x_{3}^{s}\right)=\phi_{r+1}\left(x_{I}\right) x_{3}^{s+1} .
$$

Now, we state and prove Lemma 3.2 We use Lemma 3.2 in Section 4 in the proof of Theorem 1.4.

Lemma 3.2. Let us define the polynomial $e_{k}\left(t_{1}, \ldots, t_{k}\right)$ by

$$
e_{k}\left(t_{1}, \ldots, t_{k}\right)=\left|\begin{array}{ccc}
t_{1} & \cdots & t_{k} \\
t_{1}^{2} & \cdots & t_{k}^{2} \\
\vdots & & \vdots \\
t_{1}^{2 k-1} & \cdots & t_{k}^{2^{k-1}}
\end{array}\right| .
$$

Then, in $M_{n}$, for a finite set $I=\left\{i_{1}, \ldots, i_{\ell}\right\}$ of positive integers such that $1 \leq i_{1}<$ $\cdots<i_{\ell} \leq n$, we have

$$
\begin{aligned}
\phi_{\ell}\left(x_{I}\right) & =e_{\ell}\left(x_{4 i_{1}}, \ldots, x_{4 i_{\ell}}\right) \neq 0, \\
\phi_{\ell+1}\left(x_{I}\right) & =e_{\ell}\left(x_{4 i_{1}}, \ldots, x_{4 i_{\ell}}\right)^{2} \neq 0, \\
\phi_{\ell+2}\left(x_{I}\right) & =0 .
\end{aligned}
$$


Proof. Immediately from the definition of $\partial_{j}$, we have $\partial_{j_{1}} \partial_{j_{2}}\left(x_{4 i}\right)=0$. Let $S_{\ell}$ be the set of permutations of $\{1, \ldots, \ell\}$. Let $\partial_{1}\left(x_{4 i}\right)=x_{4 i}$. Then, we have

$$
\begin{aligned}
\partial_{2} \cdots \partial_{\ell}\left(x_{I}\right) & =\sum_{\tau \in S_{\ell}}\left(\partial_{\tau(1)}\left(x_{4 i_{1}}\right)\right) \cdots\left(\partial_{\tau(\ell)}\left(x_{4 i_{\ell}}\right)\right) \\
& =\left|\begin{array}{ccc}
\partial_{1}\left(x_{4 i_{1}}\right) & \cdots & \partial_{1}\left(x_{4 i_{\ell}}\right) \\
\vdots & & \vdots \\
\partial_{\ell}\left(x_{4 i_{1}}\right) & \cdots & \partial_{\ell}\left(x_{4 i_{\ell}}\right)
\end{array}\right| \\
& =\left|\begin{array}{ccc}
x_{4 i_{1}} & \cdots & x_{4 i_{\ell}} \\
x_{4 i_{1}}^{2} & \cdots & x_{4 i_{\ell}}^{2} \\
\vdots & & \vdots \\
x_{4 i_{1}}^{2^{\ell-1}} & \cdots & x_{4 i_{\ell}}^{2-1}
\end{array}\right| \\
& =e_{\ell}\left(x_{4 i_{1}}, \ldots, x_{4 i_{\ell}}\right)
\end{aligned}
$$

as desired. Similarly, we have

$$
\partial_{2} \cdots \partial_{\ell+1}\left(x_{I}\right)=e_{\ell}\left(x_{4 i_{1}}, \ldots, x_{4 i_{\ell}}\right)^{2} .
$$

Therefore, we have

$$
\partial_{2} \cdots \partial_{\ell+2}\left(x_{I}\right)=\partial_{\ell+2}\left(e_{\ell}\left(x_{4 i_{1}}, \ldots, x_{4 i_{\ell}}\right)^{2}\right)=0 .
$$

We end this section by stating and proving Lemma 3.3. We use Lemma 3.3 in the computation of $E_{2^{r+2}}$-term in Section 5

Lemma 3.3. Let $s$ be a non-negative integer and $r$ an integer greater than or equal to 2. Let $x \in M_{n, \ell-r+1}$ be a linear combination of $\phi_{r}\left(x_{I}\right) x_{3}^{s}$, where $\ell(I)=\ell$. Suppose that $\ell-r+1+s$ is even. Suppose that $Q_{r+1}(x)=0$. Then $x$ is a linear combination of $\phi_{r+1}\left(x_{J}\right) x_{3}^{s-1}$, where $\ell(J)=\ell+1$. Moreover, if $s \geq 1$, there exists $y \in M_{n}$, which is a linear combination of $\phi_{r}\left(x_{J} x_{3}^{s-1}\right)$, such that $Q_{r+1}(y)=x$.

For $J=\left\{j_{1}, \ldots, j_{r}\right\}$ such that $2 \leq j_{1}<\cdots<j_{r}$, we denote $\partial_{j_{1}} \cdots \partial_{j_{r}}$ by $\partial_{J}$. By Proposition 3.1. Lemma 3.3 immediately follows from Proposition 3.4 below. Lemma 3.3 is the case $J=\{2, \ldots, r+1\}$ in Proposition 3.4

Proposition 3.4. Suppose that $J=\left\{j_{1}, \ldots, j_{r}\right\}, 2 \leq j_{1}<\cdots<j_{r}$ and that $i \geq 1$. Then, the homomorphism

$$
\partial_{J}: M_{n, i+r-1} \rightarrow M_{n, i} \cap\left(\bigcap_{j \in J} \operatorname{Ker} \partial_{j}\right)
$$

is surjective.

Proof. We prove the proposition by induction on $n$. First, we deal with the case $n=1$. Suppose that $x \in M_{1,1}$. Then, $x=\alpha x_{41}$ for some $\alpha \in L_{1}$. Since

$$
\partial_{j}\left(\alpha x_{41}\right)=\alpha x_{41}^{2^{j-1}}
$$

we have

$$
M_{1,1} \cap \operatorname{Ker} \partial_{j}=\{0\} .
$$

$M_{1, i}=\{0\}$ for $i \geq 2$. So, the proposition for $n=1$ is obvious. 
Next, we assume that $n \geq 2$. Suppose that $i \geq 1$ and

$$
f=f_{k, k} x_{4 n}^{k}+f_{k, k+1} x_{4 n}^{k+1}+\cdots \in M_{n, i} \cap\left(\bigcap_{j \in J} \operatorname{Ker} \partial_{j}\right),
$$

where $f_{k, \ell} \in M_{n-1}$. We assume for now that there exists an element $g_{k} \in M_{n-1}$ such that $\partial_{J}\left(g_{k}\right)=f_{k, k}$. Then,

$$
f-\partial_{J}\left(g_{k} x_{4 n}^{k}\right)=f_{k+1, k+1} x_{4 n}^{k+1}+f_{k+1, k+2} x_{4 n}^{k+2}+\cdots
$$

So, continuing this process,

$$
f-\partial_{J}\left(g_{k} x_{4 n}^{k}+g_{k+1} x_{4 n}^{k+1}+\cdots\right)=0
$$

Now, we complete the proof of the proposition by showing the existence of $g_{k}$ such that $\partial_{J}\left(g_{k}\right)=f_{k, k}$. If $k$ is odd, or if $k$ is even and $i \geq 2$, then for $j \in J$, the coefficient of $x_{4 n}^{k}$ in $\partial_{j}(f)$ is

$$
\partial_{j}\left(f_{k, k}\right)=0 .
$$

It is in $M_{n-1, i}$ if $k$ is even and in $M_{n-1, i-1}$ if $k$ is odd. So, if $k$ is even or $i \geq 2$, by the inductive hypothesis, there exists $g_{k}$ such that $\partial_{J}\left(g_{k}\right)=f_{k, k}$. If $k$ is odd and $i=1$, then the coefficient of $x_{4 n}^{k+2^{j_{1}-1}-1}$ is

$$
f_{k, k}+\partial_{j_{1}} f_{k, k+2^{j_{1}-1}-1}=0 .
$$

For $j \in\left\{j_{2}, \ldots, j_{r}\right\}$, the coefficient of $x_{4 n}^{k+2^{j_{1}-1}-1}$ in $\partial_{j}(f)$ is

$$
\partial_{j}\left(f_{k, k+2^{j_{1}-1}-1}\right)=0 .
$$

Since $f_{k, k+2^{j_{1}-1}-1}$ is in $M_{n-1,1}$, by the inductive hypothesis, there exists $g_{k}$ such that $\partial_{j_{2}} \cdots \partial_{j_{r}}\left(g_{k}\right)=f_{k, k+2^{j_{1}-1}-1}$. So, we have $\partial_{J}\left(g_{k}\right)=f_{k, k}$. This completes the proof.

\section{The Atiyah-Hirzebruch spectral Sequence}

In this section, we describe the Atiyah-Hirzebruch spectral sequence for

$$
B P\langle m\rangle^{*}\left(B G_{n}\right)
$$

for $m \geq 0$ and $n \geq 1$.

We begin with notation. Suppose that $y$ ranges over a finite subset $\{y\}$ of an $R$ module $M$. Let $R^{\prime}$ be a subring of $R$. Then, we denote by $R^{\prime}\langle y\rangle$ the $R^{\prime}$-submodule of $M$ generated by $\{y\}$. If $I \subset R^{\prime}$ is an annihilator ideal of $\{y\}$, then we write $\left(R^{\prime} / I\right)\langle y\rangle$ for $R^{\prime}\langle y\rangle$. If it is a free $\left(R^{\prime} / I\right)$-module, then we write $\left(R^{\prime} / I\right)\{y\}$ or $\left(R^{\prime} / I\right)\langle y\rangle$ for it. If $\{y\}$ is the empty set, then we put $\left(R^{\prime} / I\right)\{y\}=\left(R^{\prime} / I\right)\langle y\rangle=\{0\}$.

Now, we compute the integral cohomology $B P\langle 0\rangle^{*}\left(B G_{n}\right)=H^{*}\left(B G_{n} ; \mathbb{Z}_{(2)}\right)$. We computed the mod 2 cohomology ring of $B G_{n}$ in Section 2 and we obtained

$$
H^{*}\left(B G_{n} ; \mathbb{Z} / 2\right)=\mathbb{Z} / 2\left[x_{2}^{2}, x_{3}^{2}, x_{41}, \ldots, x_{4 n}\right]\left\{1, x_{2}, x_{3}, x_{2} x_{3}\right\} .
$$

The Bockstein homomorphism $Q_{0}$ maps $x_{2}^{2}, x_{3}^{2}, x_{41}, x_{42}, \ldots, x_{4 n}$ to zero, so that $Q_{0}$ is a $\mathbb{Z} / 2\left[x_{2}^{2}, x_{3}^{2}, x_{41}, \ldots, x_{4 n}\right]$-module homomorphism. Moreover, since

$$
Q_{0}\left(x_{2}\right)=x_{3}, Q_{0}\left(x_{2} x_{3}\right)=x_{3}^{2}
$$

we have

$$
\begin{aligned}
\operatorname{Ker} Q_{0} & =\mathbb{Z} / 2\left[x_{2}^{2}, x_{41}, \ldots, x_{4 n}\right] \oplus \mathbb{Z} / 2\left[x_{2}^{2}, x_{3}^{2}, x_{41}, \ldots, x_{4 n}\right]\left\{x_{3}, x_{3}^{2}\right\}, \\
\operatorname{Im} Q_{0} & =\mathbb{Z} / 2\left[x_{2}^{2}, x_{3}^{2}, x_{41}, \ldots, x_{4 n}\right]\left\{x_{3}, x_{3}^{2}\right\}
\end{aligned}
$$


and

$$
\operatorname{Ker} Q_{0} / \operatorname{Im} Q_{0}=\mathbb{Z} / 2\left[x_{2}^{2}, x_{41}, \ldots, x_{4 n}\right] .
$$

So, the integral cohomology of $B G_{n}$ localized at 2 is

$$
\mathbb{Z}_{(2)}\left[x_{2}^{2}, x_{41}, \ldots, x_{4 n}\right] \oplus \mathbb{Z} / 2\left[x_{2}^{2}, x_{3}^{2}, x_{41}, \ldots, x_{4 n}\right]\left\{x_{3}, x_{3}^{2}\right\} .
$$

Next, we describe the $E_{2}$-term of the Atiyah-Hirzebruch spectral sequence. For the sake of notational simplicity, we write $v_{0}$ for 2 in the coefficient ring $\mathbb{Z}_{(2)}\left[v_{1}, \ldots, v_{m}\right]$ of the truncated Brown-Peterson cohomology $B P\langle m\rangle^{*}(-)$. Let

$$
\begin{aligned}
D & =\mathbb{Z}_{(2)}\left[x_{2}^{2}, x_{3}^{2}, x_{41}^{2}, \ldots, x_{4 n}^{2}, v_{1}, \ldots, v_{m}\right] /\left(v_{0} x_{3}^{2}\right), \\
C & =\mathbb{Z}_{(2)}\left[x_{2}^{2}, x_{41}^{2}, \ldots, x_{4 n}^{2}, v_{1}, \ldots, v_{m}\right] .
\end{aligned}
$$

We regard $C$ as a subring of $D$. We write $D_{r}$ for $D /\left(v_{0}, \ldots, v_{r}\right)$. We may decompose $D$ to $C\{1\} \oplus D_{0}\left\{x_{3}^{2}\right\}$. As in Section 3, for a finite set $I=\left\{i_{1}, \ldots, i_{\ell}\right\}$ of positive integers $i_{1}, i_{2}, \ldots, i_{\ell}$ such that $1 \leq i_{1}<\cdots<i_{\ell} \leq n$, we write $x_{I}$ for the monomial

$$
x_{4 i_{1}} \cdots x_{4 i_{\ell}}
$$

and $\ell(I)$ for the integer $\ell \geq 1$. The number of such sets $I$ is $2^{n}-1$ and the set $\left\{x_{I}\right\}$ is a finite set consisting of $2^{n}-1$ elements. Let $y_{0}$ range over $\left\{x_{I}\right\}$, that is, $\left\{y_{0}\right\}=\left\{x_{I}\right\}$. The $E_{2}$-term of the Atiyah-Hirzebruch spectral sequence for $B P\langle m\rangle^{*}\left(B G_{n}\right)$ is isomorphic to

$$
D\left\{1, y_{0}\right\} \oplus D_{0}\left\{x_{3}, x_{3} y_{0}\right\}=C\left\{1, y_{0}\right\} \oplus D_{0}\left\{x_{3}, x_{3} y_{0}, x_{3}^{2}, x_{3}^{2} y_{0}\right\}
$$

Next, we describe the first non-trivial differential $d_{3}$ and the $E_{4}$-term. Let $y_{1}$, $z_{1}$ range over

$$
\begin{aligned}
& \left\{x_{I} \mid \ell(I) \text { is even }\right\}, \\
& \left\{x_{I} \mid \ell(I) \text { is odd }\right\},
\end{aligned}
$$

respectively. Then, $\left\{y_{0}\right\}=\left\{y_{1}\right\} \cup\left\{z_{1}\right\}$. Since the Milnor operation $Q_{1}$ maps $x_{3}$, $x_{4 i}$ to

$$
Q_{1}\left(x_{3}\right)=x_{3}^{2}, \quad Q_{1}\left(x_{4 i}\right)=x_{3} x_{4 i},
$$

the first non-trivial differential $d_{3}$ is given by

$$
\begin{aligned}
d_{3}(1) & =0, & d_{3}\left(x_{3}\right) & =v_{1} x_{3}^{2}, \\
d_{3}\left(y_{1}\right) & =0, & d_{3}\left(x_{3} y_{1}\right) & =v_{1} x_{3}^{2} y_{1}, \\
d_{3}\left(z_{1}\right) & =v_{1} x_{3} z_{1}, & d_{3}\left(x_{3} z_{1}\right) & =0 .
\end{aligned}
$$

So, we have

$$
\begin{aligned}
\operatorname{Ker} d_{3} & =C\left\{1, y_{1}, v_{0} z_{1}\right\} \oplus D_{0}\left\{x_{3}^{2}, x_{3}^{2} y_{1}, x_{3} z_{1}\right\} \\
\operatorname{Im} d_{3} & =D_{0}\left\{v_{1} x_{3}^{2}, v_{1} x_{3}^{2} y_{1}, v_{1} x_{3} z_{1}\right\}
\end{aligned}
$$

and

$$
\text { Ker } d_{3} / \operatorname{Im} d_{3}=C\left\{1, y_{1}, v_{0} z_{1}\right\} \oplus D_{1}\left\{x_{3}^{2}, x_{3}^{2} y_{1}, x_{3} z_{1}\right\} .
$$

One may regard $C\left\{1, y_{1}, v_{0} z_{1}\right\}, D_{1}\left\{x_{3}^{2} y_{1}, x_{3} z_{1}\right\}$ as $C\left\langle 1, y_{1}, v_{0} y_{0}\right\rangle, D_{1}\left\langle x_{3}^{2} y_{1}, x_{3} z_{1}\right\rangle$, respectively. Hence, summing up the above, we have the following proposition. 
Proposition 4.1. We have

$$
\begin{aligned}
& E_{2}=D\left\{1, y_{0}\right\} \oplus D_{0}\left\{x_{3}, x_{3} y_{0}\right\}, \\
& E_{4}=C\left\langle 1, y_{1}, v_{0} y_{0}\right\rangle \oplus D_{1}\left\langle x_{3}^{2}, x_{3}^{2} y_{1}, x_{3} z_{1}\right\rangle .
\end{aligned}
$$

Now, we describe $E_{2^{r+1}}$-terms for $r \geq 2$. In Section 3 for $r \geq 2$, we defined $\phi_{r}\left(x_{I}\right)$ by

$$
\phi_{r}\left(x_{I}\right)=\partial_{2} \cdots \partial_{r}\left(x_{I}\right)
$$

in

$$
M_{n}=\mathbb{Z} / 2\left[x_{2}^{2}, x_{3}, x_{41}, \ldots, x_{4 n}\right] .
$$

By abuse of notation, we denote an element in $D\left\{1, y_{0}\right\}$ representing $\phi_{r}\left(x_{I}\right)$ in $D_{0}\left\{1, y_{0}\right\}$ by the same symbol $\phi_{r}\left(x_{I}\right)$. We also assume that $e_{r}, y_{r}, z_{r}$ range over the following finite sets

$$
\begin{aligned}
& \left\{\phi_{r}\left(x_{I}\right) \mid \ell(I)-r+1=0\right\}, \\
& \left\{\phi_{r}\left(x_{I}\right) \mid \ell(I)-r+1>0 \text { and } \ell(I)-r+1 \text { is even }\right\}, \\
& \left\{\phi_{r}\left(x_{I}\right) \mid \ell(I)-r+1>0 \text { and } \ell(I)-r+1 \text { is odd }\right\},
\end{aligned}
$$

respectively. We state our main result as follows:

Proposition 4.2. Suppose $r \geq 1$. Let $k=\min \{m, n+1\}$. For $r \leq k$, the $E_{2^{r+1}-}$ term of the Atiyah-Hirzebruch spectral sequence is

$$
E_{2^{r+1}}=C\left\langle 1, y_{r}, v_{s} y_{s}\right\rangle \oplus D_{1} /\left(v_{t} e_{t}\right)\left\{x_{3}^{2}\right\} \oplus D_{r}\left\langle x_{3}^{2} y_{r}, x_{3} z_{r}\right\rangle
$$

where $s$ ranges over $\{0, \ldots, r-1\}$ and $t$ ranges over $\{2, \ldots, r\}$. Moreover, the spectral sequence collapses at the $E_{2^{k+1}}$-level, that is, $E_{\infty}=E_{2^{k+1}}$.

Example 4.3. We compute the Brown-Peterson cohomology of $B G_{1}$.

The case $n=1, m \geq 2$ :

$$
\begin{aligned}
& \left\{y_{1}\right\}=\emptyset, \quad\left\{z_{1}\right\}=\left\{x_{41}\right\}, \\
& \left\{y_{2}\right\}=\emptyset, \quad\left\{z_{2}\right\}=\emptyset, \quad\left\{e_{2}\right\}=\left\{x_{41}^{2}\right\} \\
& E_{2}=C\left\{1, x_{41}\right\} \oplus D_{0}\left\{x_{3}^{2}, x_{3}^{2} x_{41}, x_{3}, x_{3} x_{41}\right\} \\
& E_{4}=C\left\{1, v_{0} x_{41}\right\} \oplus D_{1}\left\{x_{3}^{2}\right\} \oplus D_{1}\left\{x_{3} x_{41}\right\}, \\
& E_{8}=C\left\{1, v_{0} x_{41}\right\} \oplus D_{1} /\left(v_{2} x_{41}^{2}\right)\left\{x_{3}^{2}\right\}
\end{aligned}
$$

So, $x_{41}$ lifts up to $B P\langle 0\rangle^{*}\left(B G_{1}\right)$ that corresponds to the $E_{2}$-term above and it does not lift to $B P\langle 1\rangle^{*}\left(B G_{1}\right)$ that corresponds to the $E_{4}$-term above.

Example 4.4. We compute the Brown-Peterson cohomology of $B G_{2}$. The case $n=2, m \geq 3$ :

$$
\begin{array}{lll}
\left\{y_{1}\right\}=\left\{x_{41} x_{42}\right\}, & \left\{z_{1}\right\}=\left\{x_{41}, x_{42}\right\}, & \\
\left\{y_{2}\right\}=\emptyset, & \left\{z_{2}\right\}=\left\{x_{41}^{2} x_{42}+x_{41} x_{42}^{2}\right\}, & \left\{e_{2}\right\}=\left\{x_{41}^{2}, x_{42}^{2}\right\}, \\
\left\{y_{3}\right\}=\emptyset, & \left\{z_{3}\right\}=\emptyset, & \left\{e_{3}\right\}=\left\{x_{41}^{2} x_{42}^{4}+x_{41}^{4} x_{42}^{2}\right\} .
\end{array}
$$




$$
\begin{aligned}
E_{4} & =C\left\{1, x_{41} x_{42}, v_{0} x_{41}, v_{0} x_{42}\right\} \\
& \oplus D_{1}\left\{x_{3}^{2}\right\} \oplus D_{1}\left\{x_{41} x_{42} x_{3}^{2}, x_{3} x_{41}, x_{3} x_{42}\right\} \\
E_{8} & =C\left\langle 1, v_{1} x_{41} x_{42}, v_{0} x_{41} x_{42}, v_{0} x_{41}, v_{0} x_{42}\right\rangle \\
& \oplus D_{1} /\left(v_{2} x_{41}^{2}, v_{2} x_{42}^{2}\right)\left\{x_{3}^{2}\right\} \oplus D_{2}\left\{x_{3}\left(x_{41}^{2} x_{42}+x_{41} x_{42}^{2}\right)\right\} \\
E_{16} & =C\left\langle 1, v_{1} x_{41} x_{42}, v_{0} x_{41} x_{42}, v_{0} x_{41}, v_{0} x_{42}\right\rangle \\
& \oplus D_{1} /\left(v_{2} x_{41}^{2}, v_{2} x_{42}^{2}, v_{3}\left(x_{41}^{4} x_{42}^{2}+x_{41}^{2} x_{42}^{4}\right)\right)\left\{x_{3}^{2}\right\}
\end{aligned}
$$

So, $x_{41} x_{42}$ in $B P\langle 1\rangle^{*}\left(B G_{2}\right)$ that corresponds to the $E_{4}$-term above does not lift up to $B P\langle 2\rangle^{*}\left(B G_{2}\right)$ that corresponds to the $E_{8}$-term above.

We end this section by proving Theorems 1.1 and 1.4. These are immediate from Proposition 4.2

Proof of Theorem 1.1, Given $m$, let $n=m+1$. Then, the Atiyah-Hirzebruch spectral sequence for $B P\langle m\rangle^{*}\left(B G_{m+1}\right)$ collapses at the $E_{2^{m+1}}$-level and

$$
E_{\infty}=C\left\langle 1, y_{m}, v_{s} y_{s}\right\rangle \oplus D_{1} /\left(v_{t} e_{t}\right)\left\{x_{3}^{2}\right\} \oplus D_{m}\left\langle x_{3}^{2} y_{m}, x_{3} z_{m}\right\rangle .
$$

Let $u$ be an element in $B P\langle m\rangle^{*}\left(B G_{m+1}\right)$ representing the permanent cycle

$$
\phi_{m}\left(x_{41} \cdots x_{4, m+1}\right) \in\left\{y_{m}\right\}
$$

in the Atiyah-Hirzebruch spectral sequence. It is non-zero by Lemma 3.2, Moreover, since the corresponding element in the Atiyah-Hirzebruch spectral sequence for $B P\langle m+1\rangle^{*}\left(B G_{m+1}\right)$ supports non-trivial differential and so $u$ does not lift up to $B P\langle m+1\rangle^{*}\left(B G_{m+1}\right)$. Moreover, the reduction $\rho_{0}^{m}(u)$ is non-zero in

$$
\mathbb{Z}_{(2)}\left[x_{2}^{2}, x_{41}, \ldots, x_{4, m+1}\right]=H^{*}\left(B G_{m+1} ; \mathbb{Z}_{(2)}\right) /\left(x_{3}\right),
$$

therefore it is non-torsion.

Proof of Theorem 1.4. By Proposition 4.2 the odd degree part of the $E_{\infty}$-term is

$$
D_{r}\left\langle x_{3} z_{r}\right\rangle \text {. }
$$

However, by definition, $\left\{z_{r}\right\}=\emptyset$ if $r \geq n+1$. By Lemma3.2 $\left\{z_{r}\right\}$ contains non-zero element $\phi_{r}\left(x_{41} \cdots x_{4 r}\right)$ for $r \leq n$. Hence, $D_{r}\left\langle x_{3} z_{r}\right\rangle=\{0\}$ if and only if $r \geq n+1$. If $m \leq n$, the spectral sequence collapses at the $E_{2^{m+1}}$-level and it contains non-zero odd degree elements. If $m \geq n+1$, then $E_{2^{n+2}}$-term has no non-zero odd degree

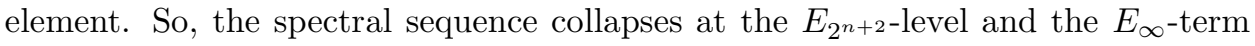
also does not have any non-zero odd degree element.

\section{Proof of Proposition 4.2}

To prove Proposition 4.2, we need to study non-trivial differentials in the spectral sequence. To this end, we prove Lemmas 5.1 and 5.2 before the proof of Proposition 4.2

We use the following Lemma 5.1 to show that the differential $d_{u}$ is trivial for $2^{r} \leq u \leq 2^{r+1}-2$.

Lemma 5.1. The following statements (1), (2) and (3) hold:

(1) For $2 \leq u<2^{r+2}-1,\left(D_{r}\left\langle x_{3}^{2} y_{r}, x_{3} z_{r}\right\rangle\right)^{*,-u+1}=\{0\}$.

(2) Suppose that $x \in C\left\langle 1, y_{r}, v_{s} y_{s}\right\rangle$ and $v_{2} x=\cdots=v_{r} x=0$. Then, we have $x=0$. 
(3) Suppose that $x \in D_{1} /\left(v_{t} e_{t}\right)\left\{x_{3}^{2}\right\}, x \equiv 0$ in $D_{r} /\left(v_{t} e_{t}\right)\left\{x_{3}^{2}\right\}=D_{r}\left\{x_{3}^{2}\right\}$ and $v_{2} x=\cdots=v_{r} x=0$. Then, we have $x=0$ in $D_{1} /\left(v_{t} e_{t}\right)\left\{x_{3}^{2}\right\}$.

Proof. Statement (1) is clear from the fact that $D_{r}^{*,-u+1}=\{0\}$ for $2 \leq u<2^{r+2}-1$. Statement (2) is immediate from the fact that $C\left\langle y_{r}, v_{s} y_{s}\right\rangle$ is a $C$-submodule of the free $C$-module $C\left\{y_{0}\right\}$. In order to prove statement (3), we consider obvious projection

$$
\pi_{j}^{i}: D_{i} /\left(v_{t} e_{t}\right)\left\{x_{3}^{2}\right\} \rightarrow D_{j} /\left(v_{t} e_{t}\right)\left\{x_{3}^{2}\right\}
$$

where $1 \leq i \leq j \leq r$. Since $t \leq r$, it is clear that

$$
D_{r} /\left(v_{t} e_{t}\right)=D_{r}=\mathbb{Z} / 2\left[x_{2}^{2}, x_{3}^{2}, x_{41}^{2}, \ldots, x_{4 n}^{2}, v_{r+1}, \ldots, v_{m}\right] .
$$

By the assumption, we have $\pi_{r}^{1}(x)=0$. Moreover, for $i=1, \ldots, r-1$, in $D_{i}$, $\left(v_{t} e_{t}\right)=\left(v_{i+1} e_{i+1}, \ldots, v_{r} e_{r}\right)$ and the kernel of $\pi_{i+1}^{i}$ is

$$
\operatorname{Ker} \pi_{i+1}^{i}=\left(v_{i+1} x_{3}^{2}\right)=D_{i} /\left(e_{i+1}, v_{i+2} e_{i+2}, \ldots, v_{r} e_{r}\right)\left\{v_{i+1} x_{3}^{2}\right\}
$$

and Ker $\pi_{i+1}^{i}$ is a free $\mathbb{Z} / p\left[v_{i+1}\right]$-module. Therefore, $v_{i+1} \pi_{i}^{1}(x)=0$ implies $\pi_{i}^{1}(x)=$ 0 . Thus, we have $\pi_{i}^{1}(x)=0$ for $i=r-1, \ldots, 2,1$ and we have the desired result.

We use the Morava K-theory in the proof of Proposition 4.2 So, we briefly recall it. The Morava K-theory $K(r+1)^{*}(X)$ of a space $X$ is a generalized cohomology theory whose coefficient ring is $\mathbb{Z} / 2\left[v_{r+1}, v_{r+1}^{-1}\right]$ and there exists a natural transformation $\psi_{r+1}: B P\langle r+1\rangle^{*}(X) \rightarrow K(r+1)^{*}(X)$ sending $v_{i}$ to 0 for $i \leq r$ and $v_{r+1}$ to $v_{r+1}$. The first and the only non-trivial differential of the Atiyah-Hirzebruch spectral sequence for $K(r+1)^{*}(B \mathbb{Z} / 2)$ is

$$
d_{2^{r+2}-1}\left(x_{1}\right)=v_{r+1} x_{1}^{2^{r+2}}=v_{r+1} Q_{r+1}\left(x_{1}\right)
$$

where $x_{1}$ is the generator of $H^{1}(B \mathbb{Z} / 2 ; \mathbb{Z} / 2)$. So, by the Künneth formula, we have Lemma 5.2 below.

Lemma 5.2. The first nontrivial differential of the Atiyah-Hirzebruch spectral sequence for $K(r+1)^{*}\left(B(\mathbb{Z} / 2)^{n+2}\right)$ is

$$
d_{2^{r+2}-1}(x)=v_{r+1} Q_{r+1}(x)
$$

for all $x$ in $H^{*}\left(B(\mathbb{Z} / 2)^{n+2} ; \mathbb{Z} / 2\right)=E_{2}^{*, 0}$ where $E_{2}^{*, *}$ is the $E_{2}$-term of the AtiyahHirzebruch spectral sequence for the Morava $K$-theory $K(r+1)^{*}\left(B(\mathbb{Z} / 2)^{n+2}\right)$.

We use Lemma 5.2 to determine the differential $d_{2^{r+2}-1}$.

Now, we prove Proposition 4.2.

Proof of Proposition 4.2. We prove Proposition 4.2 by induction on $r \geq 1$.

For $r=1$, by Proposition 4.1, the proposition holds.

Assume that $u \geq 2^{r+1}$ and

$$
E_{u}=E_{2^{r+1}}=C\left\langle 1, y_{r}, v_{s} y_{s}\right\rangle \oplus D_{1} /\left(v_{t} e_{t}\right)\left\{x_{3}^{2}\right\} \oplus D_{r}\left\langle x_{3}^{2} y_{r}, x_{3} z_{r}\right\rangle .
$$

Let us write $E^{e v e n, *}, E^{o d d, *}$ for

$$
C\left\langle 1, y_{r}, v_{s} y_{s}\right\rangle \oplus D_{1} /\left(v_{t} e_{t}\right)\left\{x_{3}^{2}\right\} \oplus D_{r}\left\langle x_{3}^{2} y_{r}\right\rangle, \quad D_{r}\left\langle x_{3} z_{r}\right\rangle,
$$

respectively. We prove $d_{u}=0$ for $u=2^{r+1}, \ldots, 2^{r+2}-2$. Since $B P\langle m\rangle^{*}$ is concentrated in even degrees, $d_{u}=0$ if $u$ is even. So, we assume that $u$ is odd. 
Then, we have

$$
\begin{aligned}
d_{u}\left(E^{e v e n, *}\right) & \subset E^{o d d, *}, \\
d_{u}\left(E^{o d d, *}\right) & \subset E^{e v e n, *} .
\end{aligned}
$$

First, we prove $d_{u}=0$ on $E^{e v e n, *}$. By Lemma 5.1 (1), we have

$$
d_{u}\left(E^{e v e n, 0}\right) \subset\left(D_{r}\left\langle x_{3} z_{r}\right\rangle\right)^{*,-u+1}=\{0\} .
$$

Since $d_{u}$ is a $B P\langle m\rangle^{*}$-module homomorphism and $D_{1} /\left(v_{t} e_{t}\right)\left\{x_{3}^{2}\right\} \oplus D_{r}\left\langle x_{3}^{2} y_{r}\right\rangle$ is generated by

$$
\left(D_{1} /\left(v_{t} e_{t}\right)\left\{x_{3}^{2}\right\} \oplus D_{r}\left\langle x_{3}^{2} y_{r}\right\rangle\right) \cap E^{e v e n, 0}
$$

as a $B P\langle m\rangle^{*}$-module, we have

$$
d_{u}\left(D_{1} /\left(v_{t} e_{t}\right)\left\{x_{3}^{2}\right\} \oplus D_{r}\left\langle x_{3}^{2} y_{r}\right\rangle\right)=\{0\} .
$$

On the other hand, on $D_{r}\left\langle x_{3} z_{r}\right\rangle$, the multiplication by $x_{3}^{2}$ is injective. Therefore, since

$$
\begin{aligned}
x_{3}^{2} d_{u}\left(C\left\langle 1, y_{r}, v_{s} y_{s}\right\rangle\right) & =d_{u}\left(x^{2} C\left\langle 1, y_{r}, v_{s} y_{s}\right\rangle\right) \\
& \subset d_{u}\left(D_{1} /\left(v_{t} e_{t}\right)\left\{x_{3}^{2}\right\} \oplus D_{r}\left\langle x_{3}^{2} y_{r}\right\rangle\right)=\{0\},
\end{aligned}
$$

we have

$$
d_{u}\left(C\left\langle 1, y_{r}, v_{s} y_{s}\right\rangle\right)=\{0\}
$$

and so $d_{u}\left(E^{\text {even }, *}\right)=\{0\}$. Next, we prove $d_{u}=0$ on $E^{\text {odd,* }}$. By Lemma 5.1 (2), (3), we have $d_{u}\left(E^{\text {odd }, *}\right) \subset D_{r}\left\langle x_{3}^{2} y_{r}\right\rangle$ and by Lemma 5.1 (1), $d_{u}\left(E^{\text {odd }, 0}\right) \subset$ $\left(D_{r}\left\langle x_{3}^{2} y_{r}\right\rangle\right)^{*,-u+1}=\{0\}$. Since $E^{o d d, *}$ is generated by $E^{\text {odd }, 0}$ as a $B P\langle m\rangle^{*}$-module, we have $d_{u}\left(E^{\text {odd }, *}\right)=\{0\}$. Thus, $d_{u}$ is trivial for $2^{r+1} \leq u \leq 2^{r+2}-2$.

Next, we compute $d_{2^{r+2}-1}$ for $r \leq k-1$. We consider homomorphisms

$$
\begin{aligned}
& \rho_{r+1}^{m}: B P\langle m\rangle^{*}\left(B G_{n}\right) \rightarrow B P\langle r+1\rangle^{*}\left(B G_{n}\right), \\
& \iota_{n}^{*}: B P\langle r+1\rangle^{*}\left(B G_{n}\right) \rightarrow B P\langle r+1\rangle^{*}\left(B(\mathbb{Z} / 2)^{n+2}\right), \\
& \psi_{r+1}: B P\langle r+1\rangle^{*}\left(B(\mathbb{Z} / 2)^{n+2}\right) \rightarrow K(r+1)^{*}\left(B(\mathbb{Z} / 2)^{n+2}\right) .
\end{aligned}
$$

We denote the induced homomorphisms among the Atiyah-Hirzebruch spectral sequences by the same symbols. Note that $\operatorname{deg} v_{r+2}=-2^{r+3}+2$. Then, the induced homomorphism $\rho_{r+1}^{m}$ between Atiyah-Hirzebruch spectral sequences for $B P\langle m\rangle^{*}\left(B G_{n}\right)$ and $B P\langle r+1\rangle^{*}\left(B G_{n}\right)$ is an isomorphism on $E_{2^{r+2}-1}^{*, w}$ for $-2^{r+3}+$ $2<w$. A nonzero element in $D_{r}\left\langle x_{3}^{2} y_{r}, x_{3} z_{r}\right\rangle$ of the $E_{2^{r+2}-1}$-term of the AtiyahHirzebruch spectral sequence for $B P\langle r+1\rangle^{*}\left(B G_{n}\right)$ is of the form

$$
v_{r+1}^{k} f
$$

where $f$ is a nonzero element in $H^{*}\left(B G_{n}\right)$. The $E_{2^{r+2}-1}$-term of the AtiyahHirzebruch spectral sequence for $K(r+1)^{*}\left(B(\mathbb{Z} / 2)^{n+2}\right)$ is equal to its $E_{2}$-term

$$
\mathbb{Z} / 2\left[v_{r+1}, v_{r+1}^{-1}\right] \otimes H^{*}\left(B(\mathbb{Z} / 2)^{n+2}\right) .
$$

The induced homomorphism $\psi_{r+1} \circ \iota_{n}^{*}$ sends $v_{r+1}^{k} f$ to $v_{r+1}^{k} \iota_{n}^{*}(f)$. By Proposition 2.2, $\iota_{n}^{*}: H^{*}\left(B G_{n}\right) \rightarrow H^{*}\left(B(\mathbb{Z} / 2)^{n+2}\right)$ is injective. So, the induced homomorphism $\psi_{r+1} \circ \iota_{n}^{*}$ is injective on $D_{r}\left\langle x_{3}^{2} y_{r}, x_{3} z_{r}\right\rangle$. Similarly, the kernel of $\psi_{r+1} \circ \iota_{n}^{*}$ on $D_{1} /\left(v_{t} e_{t}\right)\left\{x_{3}^{2}\right\}$ of the $E_{2^{r+2}-1}$-term of the Atiyah-Hirzebruch spectral sequence for $B P\langle r+1\rangle^{*}\left(B G_{n}\right)$ is the kernel of the obvious projection

$$
\pi_{r}^{1}: D_{1} /\left(v_{t} e_{t}\right)\left\{x_{3}^{2}\right\} \rightarrow D_{r}\left\{x_{3}^{2}\right\} .
$$


By Lemma 5.2 the first non-trivial differential in the Atiyah-Hirzebruch spectral sequence for the Morava $K$-theory $K(r+1)^{*}\left(B(\mathbb{Z} / 2)^{n+2}\right)$ is

$$
d_{2^{r+2}-1}(x)=v_{r+1} Q_{r+1}(x) \in E_{2^{r+2}-1}^{*,-2^{r+2}+2}
$$

for $x \in E_{2^{r+2}-1}^{*, 0}$. In the Atiyah-Hirzebruch spectral sequence for $B P\langle m\rangle^{*}\left(B G_{n}\right)$, we have

$$
d_{2^{r+2}-1}\left(E^{e v e n, 0}\right) \subset\left(D_{r}\left\langle x_{3} z_{r}\right\rangle\right)^{*,-2^{r+2}+2}
$$

and

$$
d_{2^{r+2}-1}\left(E^{o d d, 0}\right) \subset\left(D_{1} /\left(v_{t} e_{t}\right)\left\{x_{3}^{2}\right\} \oplus D_{r}\left\langle x_{3}^{2} y_{r}\right\rangle\right)^{*,-2^{r+2}+2} .
$$

Hence, we have

$$
\begin{aligned}
d_{2^{r+2}-1}\left(\phi_{r}\left(x_{I}\right)\right) & =v_{r+1} \phi_{r+1}\left(x_{I}\right) x_{3}, \\
d_{2^{r+2}-1}\left(\phi_{r}\left(x_{I}\right) x_{3}\right) & =v_{r+1} \phi_{r+1}\left(x_{I}\right) x_{3}^{2}+\alpha_{I},
\end{aligned}
$$

where $\alpha_{I}$ is in $\operatorname{Ker} \pi_{r}^{1}$. However, since

$$
v_{2} \phi_{r}\left(x_{I}\right) x_{3}=\cdots=v_{r} \phi_{r}\left(x_{I}\right) x_{3}=0
$$

and

$$
v_{2} \phi_{r+1}\left(x_{I}\right) x_{3}^{2}=\cdots=v_{r} \phi_{r+1}\left(x_{I}\right) x_{3}^{2}=0,
$$

we have $v_{2} \alpha_{I}=\cdots=v_{r} \alpha_{I}=0$. By Lemma 5.1 (3), we have $\alpha_{I}=0$. Therefore, we have

$$
\begin{aligned}
d_{2^{r+2}-1}\left(\phi_{r}\left(x_{I}\right)\right) & =v_{r+1} \phi_{r+1}\left(x_{I}\right) x_{3}, \\
d_{2^{r+2}-1}\left(\phi_{r}\left(x_{I}\right) x_{3}\right) & =v_{r+1} \phi_{r+1}\left(x_{I}\right) x_{3}^{2} .
\end{aligned}
$$

Now, we compute the $E_{2^{r+2}}$-terms. We have that

$$
\operatorname{Im} d_{2^{r+2}-1}=\left(v_{r+1} e_{r+1}\right)\left\{x_{3}^{2}\right\} \oplus D_{r}\left\langle v_{r+1} x_{3}^{2} y_{r+1}, v_{r+1} x_{3} z_{r+1}\right\rangle,
$$

where $\left(v_{r+1} e_{r+1}\right)$ is the ideal generated by $v_{r+1} e_{r+1}$ in $D_{1} /\left(v_{t} e_{t}\right)$ and $t$ ranges over $\{2, \ldots, r\}$. The kernel of the multiplication by $x_{3}^{2}$ is $C\left\langle v_{1}, v_{2}, \ldots, v_{r}, v_{r} y_{r}, v_{s} y_{s}\right\rangle$. On the other hand, by Lemma 3.3. we have

$$
\begin{array}{r}
\operatorname{Ker} d_{2^{r+1}-1} \cap\left(D_{1} /\left(v_{t} e_{t}\right)\left\{x_{3}^{2}\right\} \oplus D_{r}\left\langle x_{3}^{2} y_{r}, x_{3} z_{r}\right\rangle\right) \\
=D_{1} /\left(v_{t} e_{t}\right)\left\{x_{3}^{2}\right\} \oplus D_{r}\left\langle x_{3}^{2} y_{r+1}, x_{3} z_{r+1},\right\rangle .
\end{array}
$$

Hence, we have the required $E_{2^{r+2} \text {-term. }}$

Finally, we complete the proof by showing $d_{u}=0$ for $u \geq 2^{k+1}$. Since $k=$ $\min \{m, n+1\}$, we need to consider two cases:

Case 1. The case $k=m$. In this case, $r=k, u \geq 2^{r+1}$. Then, we have

$$
E_{u}=E^{\text {even }, *} \oplus E^{o d d, *} .
$$

Moreover, we have $D_{r}=\mathbb{Z} / 2$. On the one hand, we have

$$
\left(D_{r}\left\{x_{3}^{2}\right\}\right)^{*,-u+1}=\{0\} .
$$

So, by Lemma 5.1 (2), (3), we have $d_{u}\left(E^{o d d, *}\right) \subset D_{r}\left\langle x_{3}^{2} y_{r}\right\rangle$. It is clear that $d_{u}\left(E^{\text {even }, *}\right) \subset D_{r}\left\langle x_{3} z_{r}\right\rangle$. On the other hand, from $D_{r}=\mathbb{Z} / 2$, we have

$$
\left(D_{r}\left\langle x_{3}^{2} y_{r}, x_{3} z_{r}\right\rangle\right)^{*,-u+1}=\{0\} .
$$

Therefore, we have

$$
d_{u}\left(E_{u}\right) \subset\left(D_{r}\left\langle x_{3}^{2} y_{r}, x_{3} z_{r}\right\rangle\right)^{*,-u+1}=\{0\} .
$$


Case 2. The case $k=n+1$. In this case, as in the proof of Theorem 1.4, since $\left\{z_{k}\right\}=\emptyset$, the $E_{2^{k+1}}$-term has no odd degree generators. Therefore, the spectral sequence collapses at the $E_{2^{k+1}}$-level.

Acknowledgement. The author would like to thank the anonymous referee for his or her advice. It was helpful in improving the presentation of this paper.

\section{REFERENCES}

[AH62] M. F. Atiyah and F. Hirzebruch, Analytic cycles on complex manifolds, Topology 1 (1962), 25-45, DOI 10.1016/0040-9383(62)90094-0. MR0145560

[Ant16] B. Antieau, On the integral Tate conjecture for finite fields and representation theory, Algebr. Geom. 3 (2016), no. 2, 138-149, DOI 10.14231/AG-2016-007. MR3477951

[CTS10] J.-L. Colliot-Thélène and T. Szamuely, Autour de la conjecture de Tate à coefficients $\mathbf{Z}_{\ell}$ pour les variétés sur les corps finis, The geometry of algebraic cycles, Clay Math. Proc., vol. 9, Amer. Math. Soc., Providence, RI, 2010, pp. 83-98 (French). MR2648666

[Kam15] M. Kameko, On the integral Tate conjecture over finite fields, Math. Proc. Cambridge Philos. Soc. 158 (2015), no. 3, 531-546, DOI 10.1017/S0305004115000134. MR3335426

[KY93] A. Kono and N. Yagita, Brown-Peterson and ordinary cohomology theories of classifying spaces for compact Lie groups, Trans. Amer. Math. Soc. 339 (1993), no. 2, 781-798, DOI 10.2307/2154298. MR1139493

[Lan72] P. S. Landweber, Elements of infinite filtration in complex cobordism, Math. Scand. 30 (1972), 223-226, DOI 10.7146/math.scand.a-11077. MR0326762

[PY15] A. Pirutka and N. Yagita, Note on the counterexamples for the integral Tate conjecture over finite fields, Doc. Math. Extra vol.: Alexander S. Merkurjev's sixtieth birthday (2015), 501-511. MR3404393

[Qui16] G. Quick, Examples of non-algebraic classes in the Brown-Peterson tower (2016), available at arXiv:1611.09136

[SV05] C. Soulé and C. Voisin, Torsion cohomology classes and algebraic cycles on complex projective manifolds, Adv. Math. 198 (2005), no. 1, 107-127, DOI 10.1016/j.aim.2004.10.022. MR2183252

[Tot97] B. Totaro, Torsion algebraic cycles and complex cobordism, J. Amer. Math. Soc. 10 (1997), no. 2, 467-493, DOI 10.1090/S0894-0347-97-00232-4. MR1423033

[Yag05] N. Yagita, Applications of Atiyah-Hirzebruch spectral sequences for motivic cobordism, Proc. London Math. Soc. (3) 90 (2005), no. 3, 783-816, DOI 10.1112/S0024611504015084. MR2137831

Department of Mathematical Sciences, Shibaura Institute of Technology, 307 MinumaKu Fukasaku, Saitama-City 337-8570, JaPAN

E-mail address: kameko@shibaura-it.ac.jp 